\title{
Effect of a 12-week Resistance-exercise Training Program on the Physical Self-perceptions of Children
}

\author{
Margaret T. Jones $^{1 *}$ and Brook L. Skidmore ${ }^{2}$ \\ ${ }^{1}$ George Mason University, Fairfax, VA, USA \\ ${ }^{2}$ University of Western Washington, Bellingham, WA, USA
}

Received: April 17, 2015; Accepted: May 28, 2015; Published: July 16, 2015

*Corresponding author: Margaret T. Jones, Ph.D., George Mason University, SMART Laboratory,Division of Health and Human Performance, 10900 University Blvd, MS 4E5, Manassas, VA 20110-2203,USA; Tel: 703-993-3247; FAX: 703-993-2025; E-mail: mjones15@gmu.edu

\begin{abstract}
Resistance-exercise training improves self-concept in adolescents and adults, while middle childhood (8-10 year) remains understudied. The purpose was to analyze if physical self-perceptions would change following 12-week of resistance exercise. Boys ( $\mathrm{n}=11,9.0 \pm 0.9$ year) and girls ( $n=13,9.3 \pm 0.7$ year) were randomly assigned by sex into control (CON, $n=12$ ) or experimental (EXP, $n=12$ ) groups. Week 1 pre-testing consisted of the Physical Self-Perception Profile for Children (C-PSPP), which contains 6 subscales: Global General Self-Worth (GSW), Global Physical Self-Worth (PSW), Perceived Sport Competence (SPORT), Perceived Bodily Attractiveness (BODY), Perceived Physical Strength (STRENGTH), Perceived Level Of Physical Conditioning (CONDITION). After familiarization, the EXP received 12-week of resistance exercise 2 days/week. Each exercise session consisted of: 5-min warm up, 5-min static stretching, 25-min resistance exercise, 5-min cool down, and 5-min static stretching. C-PSPP post-testing occurred after completion of the training program. A 2 × 2 repeated measures multivariate analyses of variance was used. Bonferroni pairwise post hoc analyses examined differences in the C-PSPP subscales. Alpha level was $p<0.05$. CON and EXP C-PSPP pretests were not different $(p>0.05)$. A C-PSPP post-test interaction $(p=0.037)$ existed between CON and EXP. Post hoc analyses showed gains $(p<0.05)$ for EXP in all subscales. Only SPORT improved for the CON group $(p=0.02)$. Regular resistance exercise improved all subscale scores, not just STRENGTH, in the EXP. Children who participate in resistance-exercise training display enhanced physical self-perceptions, and may have an increased awareness of their own levels of physical conditioning and strength.
\end{abstract}

Keywords: Health; Fitness; Middle childhood; Psychosocial; Strength

\section{Introduction}

There are positive health factors associated with regular exercise, yet the level of physical activity in children has continued to decline, [1,2] and childhood obesity remains a global health concern $[3,4]$. In the United States, obesity has doubled in children and tripled in adolescents over the past 30 years, with more than one third of children and adolescents currently overweight or obese [5]. Increasing physical activity in children is recommended because the early development of habitual exercise patterns appears to carry into adulthood $[2,6]$.

Despite continued reluctance by some to implement resistance-exercise training over concerns of potential injury to the developing musculoskeletal system, evidence has demonstrated both the safety and efficacy of youth resistance exercise [6-9]. Consistent resistance-exercise training in children has been reported to increase bone mineral density $[8,10,11]$ and elicit favorable changes in body composition [12-14]. Additionally, improving muscular strength in youth, who are deficient, can result in raised motor skill competence [15], and a subsequent increase in physical activity [6].

Psychological benefits of regular cardiovascular exercise [16], resistance training exercise $[17,18]$, or a combined program of cardiovascular and resistance exercise [19] have been studied in adults. Resistance-exercise training has the potential to promote positive social interactions, enhance mental health and well being, and to stimulate a positive attitude toward lifetime physical activity [16]. Such exercise for children may be related to aspects of psychological development, and the psychosocial health benefits observed in adult populations may be similar in children $[20,21]$. The extent to which resistance-exercise training may affect psychosocial status in children and adolescents, and in particular those that are overweight, remains inconclusive [22].

Perceptions of competence, self-worth, and self-efficacy are an integral part of motivation and considered to be strong predictors of physical activity in youth $[4,23]$. Significant improvements in self-efficacy have been noted in girls (age: 9-12 years) who participated in a 12-week physical activity program that incorporated resistance-exercise training, aerobic exercise, and behavioral skills training [24]. Mood and self-appraisal improved in boys and girls (age: 9-12 years) following a 12week Youth Fit for Life protocol, in both an after-school format as well as a physical education format [25]. Compared to a typical school-based physical activity program, Hispanic adolescents who participated in a 12-week structured resistance-exercise training program demonstrated significant improvements in self-concept [26]. It has also been suggested that regular physical activity may enhance self-esteem and foster self worth in early adolescent girls [27]. 
One such method of examining the self-esteem of children in relation to physical and global general self-worth is the Physical Self-Perception Profile for Children (C-PSPP), which distinguishes between low and high physical self-perception [28]. The C-PSPP, of which the basis for development was the educationallyoriented hierarchical model of self-concept presented by Shavelson et al. [29], has been used to assess physical selfperceptions in high school athletes [30] as well as in conjunction with physical activity measures in British and Russian youth [31], Canadian youth [32], and overweight/obese children from 8-12 years of age [4]. Information obtained from the use of the C-PSPP has been found to be valid and reliable, in addition to serving as a useful tool when designing physical activity programs to address low physical self-esteem in children $[28,33]$.

Resistance-exercise training in middle childhood (age: 8-10 years), like other forms of exercise, may provide positive psychological advantages that elicit feelings of competency, and aid in development as children mature physically and psychologically $[7,8,21]$. Since resistance-exercise training is an activity not commonly utilized during middle childhood, research is warranted to determine if such programs adapted to the needs of children could enhance their physical self perceptions and general self-worth.

\section{Objective}

In summary, resistance-exercise training has been shown to have a positive influence on physical and general self worth in adults and adolescents. Yet, middle childhood (8-10 year) remains relatively understudied in this area. Therefore, the objective of the current study was to analyze whether or not physical selfperceptions of children would change following a 12-week resistance-exercise training program. It was hypothesized that a resistance-exercise training program would produce higher ratings of the Global General Self-Worth subscale, Global Physical Self-Worth subscale, and four subdomain self-perceptions: Perceived Sport Competence, Perceived Bodily Attractiveness, Perceived Physical Strength, and Perceived Level of Physical Conditioning compared to the control group.

\section{Methods}

\section{Participants}

The 24 participants were boys ( $n=11$; age $X=9.0 \pm 0.9$ year) and girls ( $n=13$; age $X=9.3 \pm 0.7$ year) who were members in the same daily, after school program. Children were randomly assigned by sex into the control (CON, $\mathrm{n}=12$; 5 boys, 7 girls) or experimental (EXP, $\mathrm{n}=12 ; 6$ boys, 6 girls) groups. Institutional approved consent and medical clearance forms were used to obtain written informed consent from all parents/guardians of the participants. The Institutional Review Board for Human Subjects approved all study procedures. Familiarization and testing sessions were conducted in the facility where the children participated in the after school program.

\section{Experimental Design}

Children between the ages of 8 and 10, from the same daily, after school program, voluntarily signed up to serve as participants in the current study. Following random assignment by researchers to either the CON or EXP group, all participants completed the pre-test measure of the C-PSPP, which was administered by the researchers. However, participants were not notified of their group assignment until after completion of pretesting. The EXP group participated in a twice weekly, 12-week resistance-exercise training program at the facility of the after school program, while the CON group participated in their usual after school programming activities (e.g. games, group activities, crafts) during the same time. In both the EXP and CON groups children received individual and guided instruction as well as verbal encouragement for all activities in which they were participating. Post-testing of the C-PSPP for both groups took place following completion of the 12-week program.

\section{Physical self-perception}

Physical self-perceptions were assessed using the C-PSPP [28]. The C-PSPP contains a hierarchical ordering of factors: Global General Self-Worth (GSW) subscale, Global Physical SelfWorth (PSW) subscale, and four subdomain self-perceptions: Perceived Sport Competence (SPORT), Perceived Bodily Attractiveness (BODY), Perceived Physical Strength (STRENGTH), and Perceived Level of Physical Conditioning (CONDITION). The C-PSPP was adapted from the previously developed Physical Self Perception Profile (PSPP) [34]. The C-PSPP uses the same constructs as the PSPP but with age-appropriate language for use with child populations. It has been shown to have a high level of internal consistency and to be valid and reliable for use with child populations [28,33].

\section{Resistance-exercise training program}

After a familiarization session with the child-sized, pinloaded, resistance training machines (Hoist Fitness, San Diego, CA, USA), the EXP group received 12-week of resistance exercise 2 days/week with exercise compliance set at $\geq 80 \%$ for each individual over the course of the study. Each training session was supervised by the researchers, lasted approximately $45 \mathrm{~min}$, and consisted of: 5-min walking, biking, or stepping warm up; 5-min static stretching; $25 \mathrm{~min}$ of resistance exercise; 5-min walking, biking, or stepping cool down; and 5-min static stretching. The resistance-exercise training program consisted of 2 sets $\times 15$ repetitions (reps) of 7 exercises (Table 1 ) performed at a 3:0:2 tempo. Rest periods of 30 seconds followed the completion of each resistive exercise set. Child to instructor ratio was $4: 1$, and personal instruction was provided at every training session. The appropriate exercise intensity was determined during the familiarization session, by having participants start with the lowest setting on each child-sized machine and progress by one weight plate until the exercise could not be properly completed with good form at the selected tempo. Once the appropriate machine pin-setting was determined, participants continued to lift at that weight until 15 reps could be completed with ease at which point the weight was increased for their next training session.

\section{Statistical analysis}

Descriptive statistics (mean $\pm S E$ ) were computed for pre- 
Table 1: Resistance-exercise training program.

\begin{tabular}{|l|c|c|}
\hline $\begin{array}{l}\text { Exercise } \\
\text { Warm up: walk, bike, } \\
\text { or step }\end{array}$ & \multicolumn{1}{|c|}{ Volume } & Tempo \\
\hline Whole body stretching & 5 minutes & \\
\hline Pin-loaded machine: & $2 \times 15$ repetitions & $\begin{array}{c}\text { 3:0:2 (eccentric: 0: } \\
\text { concentric) }\end{array}$ \\
\hline 1) Chest Press & & \\
\hline 2) Latissimus Pull down & & \\
\hline 3) Leg Press & & \\
\hline 4) Leg Curl & & \\
\hline 5) Shoulder Press & & \\
\hline 6) Bicep Curl & & \\
\hline 7) Triceps Press & & \\
\hline $\begin{array}{l}\text { Cool down: walk, bike, } \\
\text { or step }\end{array}$ & 5 minutes & \\
\hline Whole body stretching & 5 minutes & \\
\hline
\end{tabular}

Table 2: Correlation between PSW and GSW global subscales for pre and post 12-week resistance-exercise training period.

\begin{tabular}{|l|c|c|}
\hline Group & Pre-training & Post-training \\
\hline Experimental $(\mathrm{n}=12)$ & $0.794^{* *}$ & $0.926^{* *}$ \\
\hline Control $(\mathrm{n}=12)$ & $0.705^{*}$ & $0.792^{* *}$ \\
\hline
\end{tabular}

** Correlation is significant at the $p<0.01$ level.

* Correlation is significant at the $p<0.05$ level.

Physical Self-Worth (PSW); General Self-Worth (GSW)

and post- test scores of each of the six C-PSPP subscales. A $2 \times 2$ repeated measures Multivariate Analyses Of Variance (MANOVA) was calculated. The Wilk's Lambda was used to test the significant difference of mean vectors of the six subscales. The six subscales of the C-PSPP were the dependent variables [28] and group (EXP, CON) served as the independent variable. Homogeneity of variance was met for all analyses as determined by Levene's test. Bonferroni pairwise post hoc analyses examined differences in the subscales of the C-PSPP. Bivariate (Pearson) correlations were computed to determine significant relationships between GSW and PSW. Strong correlations were considered to be $\mathrm{R}$-values between 0.71 and 0.90 . All statistical procedures were conducted using the Statistical Package for the Social Sciences (IBM SPSS Statistics 21.0, IBM Corporation, USA). The alpha level was set at $p<0.05$.

\section{Results}

The mean vectors for the interaction of treatment condition and test periods was significant (Wilk's $\lambda=0.491, \mathrm{~F}_{6,17}=2.932, \mathrm{p}$ $=0.38$ ) with the interaction explaining $50.9 \%$ of the variability. Post hoc analyses showed significant gains $(p<0.05)$ for EXP in all 6 subscales from pre to post. Only the SPORT subscale improved for the CON group ( $p=0.02$ ).

Mean scores for C-PSPP variables STRENGTH, BODY, and CONDITION are presented in Figure 1. No group differences existed between C-PSPP pretest subscale scores for CON and EXP $(p>0.05)$. Time $(p<0.05)$ and group effects were observed as a result of the resistance-exercise training program with the EXP group scoring higher on posttest C-PSPP subscales of STRENGTH $(p=0.005)$, BODY $(p=0.019)$, and CONDITION $(p=0.039)$.

Mean scores for C-PSPP variables SPORT, PSW, and GSW are presented in Figure 2. Time $(p<0.05)$ and group effects were observed for the EXP group on the C-PSPP subscales of PSW $(\mathrm{p}=0.049)$ and GSW $(p=0.031)$. A time effect $(p=0.003)$ was observed for the C-PSPP subscale of SPORT, but there was no group effect $(p=0.373)$.

Correlations between GSW and PSW were calculated for both groups pre- and post- training program and are presented in Table 2. Pre-training scores of GSW and PSW were positively correlated for both the EXP ( $\mathrm{r}=0.794, p<0.01)$ and CON $(\mathrm{r}=$ $0.705, p<0.05$ ) groups. Post-training GSW and PSW scores were strongly correlated for EXP $(\mathrm{r}=0.926, p<0.01)$ and CON $(r=$ 0.792, $p<0.01$ ) groups.

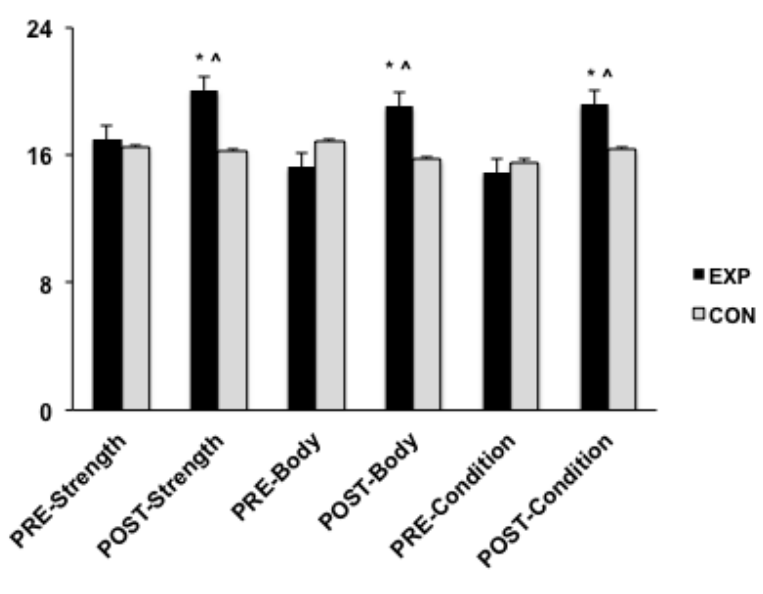

`Time effect $(p<0.05),{ }^{\wedge}$ Group effect $(p<0.05)$

Figure 1: Pre and post C-PSPP subscale scores for strength, body, and condition for experimental $(n=12)$ and control $(n=12)$ groups.

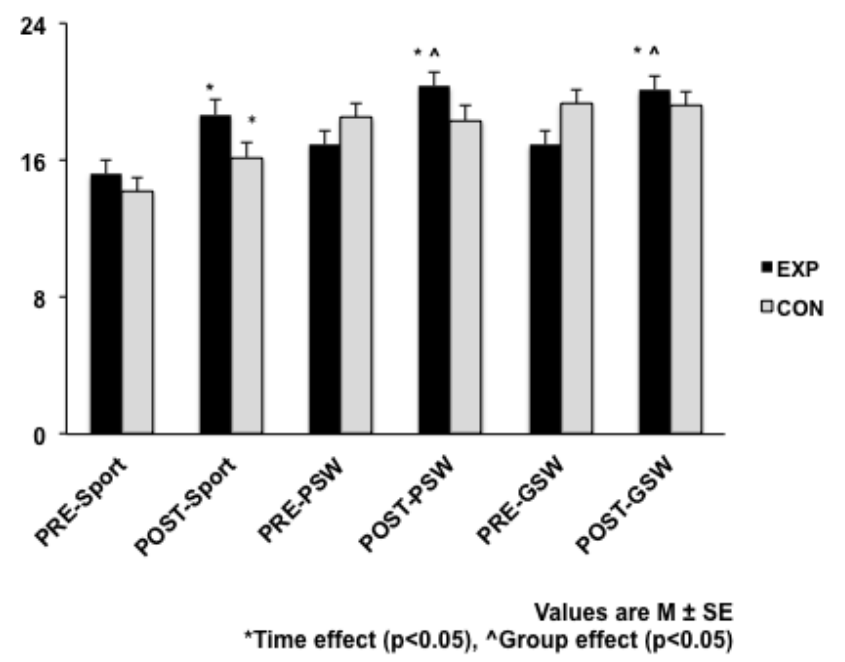

Figure 2: Pre and post C-PSPP subscale scores for sport, PSW, and GSW for experimental $(n=12)$ and control $(n=12)$ groups. 


\section{Discussion}

This is the first study designed to examine differences in ratings of C-PSPP following a 12-week, resistance-exercise training program in children of middle childhood age. The overall findings supported the hypothesis that resistance-exercise training would elicit higher C-PSPP ratings of the global subscales of GSW and PSW compared to the CON group. Additionally, three of the four subdomain self-perceptions of BODY, STRENGTH, and CONDITION were higher in the EXP group following training.

Previous research findings have demonstrated a positive psychosocial effect of physical activity. Improved ratings of physical self-perceptions in relation to physical activity, which was determined by a 7-day recall questionnaire, were noted in preadolescent [32] and adolescent [31] aged children. Improvement in perceived physical self worth, physical conditioning, and bodily attractiveness were shown in overweight/obese children following 8-week of physical activity, which was documented through the use of accelerometers [4]. Lastly, the effect of 12-week resistance training on physical selfperceptions in Hispanic adolescents resulted in increased ratings of physical and general self-worth [26].

The results from the current study are supported by what has been demonstrated previously in relation to physical activity $[31,32]$ and resistance training [26] in other age groups. The CON and EXP groups began the study with comparable pre-test C-PSPP scores. At the conclusion of the 12-week, resistanceexercise training program, the post-test C-PSPP subscale means of GSW and PSW, and the subdomain self-perceptions of BODY, CONDITION, and STRENGTH were significantly greater for the EXP group. The CON group maintained similar C-PSPP scores from pre- to post-testing with the exception of an increase in the subscale score of SPORT. This change may have been related in part to normal physical maturation. Also, SPORT C-PSPP scores may have increased as a result of the regular participation in the daily, after school program, which provided opportunities for group games, sport activity participation, and the seated activities of arts and crafts. Both CON and EXP groups participated in the aforementioned activities on a regular basis. However, the EXP group received resistance-exercise training in place of the after school program activities twice weekly.

Proposed models of the hierarchical theories of selfperception suggest that multiple components contribute to self-esteem or global general self-worth, and that physical selfperception is a component of self-esteem [28,34]. Whitehead [28] reported a significant $(p<0.001)$ correlation between PSW and GSW for both boys (0.60) and girls (0.62) in a large sample ( $n=459$ ). Additionally, the relationship between PSW and GSW was stronger than between GSW and the four subscales [28]. In the current study, the positive bivariate (Pearson) correlation between GSW and PSW increased following training (Pre: 0.795; Post: 0.926 ), as did the physical self-perception scores for the EXP group, thereby supporting the aforementioned hierarchical theory $[28,33]$. A much smaller change was observed in the positive bivariate (Pearson) correlation between GSW and PSW for the CON group from pre- to post- test (Pre: 0.705; Post: 0.792). Thus, the association between GSW and PSW appeared to strengthen as a result of the resistance exercise intervention.

Physical self- perceptions contribute in part to general selfworth. The enhancement of self-esteem is a common objective of physical activity and exercise programs [4,26,34]. Resistanceexercise training, like other forms of exercise and physical activity, has been shown to offer positive psychological benefits as well as physical benefits in both adults $[17,18]$ and adolescents [26].

Components of physical self-perceptions were most likely altered by the ability of the 12 -week, resistance-exercise training program to improve strength and increase body awareness, as has been previously suggested $[7,8]$. Although not specifically measured, children in the EXP group were observed by the researchers to have made strength gains and improved physical coordination. Improvements in strength and coordination have been demonstrated in other segments of the population for time periods comparable to the one in the present study $[19,24,26]$. Resistance training is a specific exercise activity and the primary purpose is to enhance strength. Strength is one component of physical self-perception, yet the results indicate that the net influence of 12-week of resistance-exercise training altered three of the four components of the physical self-perception (i.e., BODY, STRENGTH, CONDITION), and may have a combined positive relationship to the global general self-worth.

The current study establishes a psychosocial benefit to resistance-exercise training during middle childhood and supports the hierarchical theory of self-esteem. The 12-week, resistance-exercise training program may have also influenced other areas of the global self-esteem that are not part of physical self-perception. Resistance-exercise training may enhance areas of a child's self-esteem that were not measured by the C-PSPP, and may provide positive psychological advantages that aid in a child's physical and psychological development. Children who participate in resistance-exercise training programs may have an increased awareness of physical conditioning and muscular strength. The result of such activity may be an enhanced perception of competence, self-worth, and self-efficacy, all of which are an integral part of motivation and considered to be strong predictors of physical activity in youth [23].

We acknowledge some study limitations. First, all children were participants in the same daily after school program, which included a variety of activities like games, crafts, and sports; therefore, all had a similar base level of physical activity. Thus, results may vary in other children who are not part of this type of structured program and are less physically active. Second, muscular strength was not assessed in the control group, and was estimated once for the experimental group during the familiarization for the purpose of weight selection in subsequent workouts. Pre and post measures of muscular strength in CON and EXP groups would enable researchers to document strength gains and study them in relation to self-perception scores. Additionally, children in the current study trained twice a week for 12 weeks 
using specialized child-sized equipment for resistance training. This amount of time has been shown to elicit strength changes in other populations $[18,26]$, but a shorter training period may be effective [14]; therefore, research is warranted that examines the relationship between length of resistance-exercise training program and C-PSPP scores in middle childhood. Further, the evaluation of other exercise training programs that are less structured and, perhaps, more typical of middle childhood activity, such as a combination of aerobic and strength exercise, would be of interest. Finally, it would have been of interest to conduct a subsequent C-PSPP assessment of all participants following completion of the after school program at the end of the academic year in order to determine what, if any, changes occurred once the program ended.

\section{Conclusion}

The results of this study suggest the following in boys and girls of middle childhood age:

1) Resistance exercise training not only altered selfperceptions of physical strength, but also those of bodily attractiveness and physical conditioning for participants.

2) Resistance exercise training offers positive physical and psychosocial benefits.

\section{Acknowledgements}

The authors would like to thank all of the children who served as participants in this study. The assistance of Brian Passon, research graduate assistant, and the after school program staff was also most appreciated.

\section{References}

1. Roetert PE. The lack of childhood activity in the United States. Strength Cond J. 2004; 26(2):22-3.

2. Watts K, Jones T, Davis EW, Green D. Exercise training in obese children and adolescents: Current concepts. Sports Med 2005; 35(5):375-392.

3. Fairclough SJ, Boddy LM, Ridgers ND, Stratton G. Weight status associations with physical activity intensity and physical selfperceptions in 10- to 11-year-old children. Pediatr Exerc Sci 2012; 24:100-12.

4. Goldfield GS, Mallory R, Parker T, Cunningham T, Legg C, Lumb A, et al. Effects of modifying physical activity and sedentary behavior on psychosocial adjustment in overweight/obese children. J Pediatr Psychol 2007; 32(7):783-793.

5. Ogden CL, Carroll MD, Kit BK, Flegal KM. Prevalence of childhood and adult obesity in the United States, 2011-2012. J Am Med Assoc 2014; 311(8):806-14.

6. Faigenbaum AD, Lloyd RS, Myer GD. Youth resistance training: past practices, new perspectives, and future directions. Pediatr Exerc Sci 2013; 25:591-604

7. Faigenbaum AD, Kraemer WJ, Blimkie CJ, Jeffreys I, Micheli LJ, Nitka $\mathrm{M}$, et al. Youth resistance training: Updated position statement paper from the National Strength and Conditioning Association. J Strength Cond Res. 2009; 23:S60-S79. doi: 10.1519/JSC.0b013e31819df407.

8. Behm DG, Faigenbaum AD, Falk B, Klentrou P. Canadian Society for Exercise Physiology position paper: resistance training in children and adolescents. Appl Physio Nut Metab. 2008; 33(3):547-61. doi: 10.1139/H08-020.

9. Behringer M, vom Heede A, Yue Z, Mester J. Effects of resistance training in children and adolescents: A meta-analysis. Pediatrics 2010; 126(5):e1199-e1210. doi: 10.1542/peds.2010-0445

10.Vincent-Rodriguez G. How does exercise affect bone development during growth? Sports Med. 2006; 36(7):561-9.

11. Yu CC, Sung RY, So RC, Lui KC, Lau W, Lam PK, et al. Effects of strength training on body composition and bone mineral content in children who are obese. J Strength Cond Res. 2005; 19(3):667-72.

12. Benson AC, Torode ME, Fiatarone Singh MA. The effect of highintensity progressive resistance training on adiposity in children: A randomized controlled trial. Int J Obesity (Lond). 2008; 32(6):10161027. doi: 10.1038/ijo.2008.5.

13. Davis JN, Ventura EE, Shaibi GQ, Byrd-Williams CE, Alexander $\mathrm{KE}$, Vanni $\mathrm{AK}$, et al. Interventions for improving metabolic risk in overweight Latino youth. Int J Pediatr Obes. 2010; 5(5):451-5. doi: 10.3109/17477161003770123.

14. McGuigan MR, Tatasciore M, Newton RU, Pettigrew S. Eight weeks of resistance training can significantly alter body composition in children who are overweight or obese. J Strength Cond Res. 2009; 23(1):80-5.

15. Faigenbaum AD, Myer GD. Pediatric resistance training: Benefits, concerns and program design considerations. Curr Sports Med Rep. 2010; 9(3):161-8. doi: 10.1249/JSR.0b013e3181de1214.

16. Hassmen P, Koivula N, Uutela A. Physical Exercise and Psychological Well-Being: A Population Study in Finland. Prev Med 2000; 30(1):1725 .

17.Annesi JJ, Westcott WL. Relations of physical self-concept and muscular strength with resistance Exercise-induced feeling state scores in older women. Percept Motor Skill 2007; 104(1):183-90.

18. Jones MT, Matthews TD, Murray M, Van Raalte J, Jensen BE. Psychological correlates of performance in female athletes during a 12-week off-season strength and conditioning program. J Strength Cond Res. 2010; 24(3):619-28. doi: 10.1519/JSC.0b013e3181cc23c3.

19. Annesi J, Westcott WL, Gann S. Preliminary evaluation of a $10-w k$. resistance and cardiovascular exercise protocol on physiological and psychological measures for a sample of older women. Percept Motor Skills. 2004; 98(1):163-70.

20.Sadres E, Eliakim A, Constantini N, Lidor R, Falk B. The effect of long-term resistance training on anthropometric measures, muscle strength, and self concept in pre-pubertal boys. Pediatr Exerc Sci. 2001; 13(4):357-72.

21. Yu CC, Sung RY, Hau KT, Lam PK, Nelson EA, So RC. The effect of diet and strength training on obese children's physical self-concept. J Sports Med Phys Fitness. 2008; 48(1):76-82.

22.Schranz N, Tomkinson G, Olds T. What is the effect of resistance training on the strength, body composition, and psychosocial status of overweight and obese children and adolescents? A systematic review and meta-analysis. Sports Med. 2013; 43(9):893-907. doi: 10.1007/ s40279-013-0062-9.

23. Weiss M. Back to the Future: Research trends in youth motivation and physical activity. Pediatr Exerc Sci. 2013; 25(4):561-72.

24. Annesi JJ, Westcott WL, Faigenbaum AD, Unruh JL. Effects of a 12-week physical activity protocol delivered by YMCA afterschool counselors (Youth Fit for Life) on fitness and self-efficacy changes in 5-12 year old boys and girls. Res Q Exercise Sport 2005; 76(4):468-76. 
25. Annesi JJ, Faigenbaum AD, Westcott WL. Effects of the Youth Fit for Life protocol on physiological, mood, self-appraisal, and voluntary physical activity changes in African American preadolescents: Contrasting after-school care and physical education formats. Int J Clin Health Psychol 2007; 7(3):641-59.

26.Velez A, Golem DL, Arent SM. The impact of a 12-week resistance training program on strength, body composition, and self-concept of Hispanic adolescents. J Strength Cond Res. 2010; 24(4):1065-73.

27. Schmalz DL, Deane GD, Birch LL, Davison KK. A longitudinal assessment of the links between physical activity and self-esteem in early adolescent non-Hispanic females. J Adolesc Health. 2007; 41(6):559-65

28. Whitehead JR. A study of children's physical self-perceptions using and adapted physical self-perception profile questionnaire. Pediatr Exerc Sci. 1995; 7(2):132-51

29. Shavelson, RJ, Hubner, JJ, Stanton, GC. Self-concept: Validation of construct interpretations. Rev Educ Res. 1976; 46:407-41.

30. Welk GJ, Corbin CB, Lewis LA. Physical self-perceptions of high school athletes. Pediatr Exerc Sci. 1995; 7(2):152-61.

31. Hagger M, Ashford B, Stambulova N. Russian and British children's physical self-perceptions and physical activity participation. Pediatr Exerc Sci. 1998; 10(2):137-52.

32. Crocker PR, Eklund RC, Kowalski KC. Children' s physical activity and physical self-perceptions. J Sports Sci. 2000; 18(6): 383-94.

33. Raustorp A, Stahle A, Gudasic H, Kinnunen A, Mattsson E. Physical activity and self-perception in school children assessed with the Children and Youth-Physical Self Perception Profile. Scand J Med Sci Sports. 2005; 15(2):126-34.

34. Fox KR, Corbin CB. The physical self-perception profile: Development and preliminary validation. J Sport Exerc Psychol. 1989;11(4): 408-30. 